\title{
The [Ne III]/[Ne II] line ratio in NGC 253.
}

\author{
Daniel Devost*, Bernhard R. Brandl ${ }^{\dagger}$, L. Armus**, D. J. Barry*, G. C. \\ Sloan*, Vassilis Charmandaris*, Henrik Spoon*, Jeronimo Bernard-Salas* \\ and James R. Houck* \\ ${ }^{*}$ Center for Radiophysics and Space Research, Cornell University, 219 Space Sciences Building, \\ Ithaca, NY 14853-6801, USA \\ ${ }^{\dagger}$ Sterrewachet Leiden, PO Box 95132300 RA Leiden, The Netherlands \\ ${ }^{* *}$ Spitzer Science Center, MS 220-06, California Institute of Technology, Pasadena, CA, 91125
}

\begin{abstract}
We present results of the mapping of the nucleus of the starburst galaxy NGC 253 and its immediate surroundings using the Infrared Spectrograph ${ }^{1}$ on board the Spitzer Space Telescope. The map is centered on the nucleus of the galaxy and spans the inner $800 \times 688 \mathrm{pc}^{2}$. We perform a brief investigation of the implications of these measurement on the properties of the star formation in this region using theories developed to explain the deficiency of massive stars in starbursts.
\end{abstract}

\section{INTRODUCTION}

NGC 253 is a well studied nearby starburst galaxy located at a distance of $2.5 \mathrm{Mpc}$ [1] and is mainly powered by an episode of central star formation. This episode is generally believed to be caused by material brought to the center of the galaxy by a bar which is clearly visible in the 2MASS image [2]. The presence of a bar from both morphological and kinematic evidence, and its role as starburst trigger has been extensively discussed in the literature $([3,4,5])$.

Observations in the mid-infrared from space by the Infrared Space Observatory (ISO) were used to identify fine structure lines, molecular hydrogen lines and emission from polycyclic aromatic hydrocarbons (PAH) $[6,7,8,9]$. These papers mainly studied the nuclear region of the galaxy with the Short Wavelength Spectrograph (SWS) and ISOCAM. [7]

Since the $\mathrm{Ne}$ atom and $\mathrm{Ne}^{+}$ion have an ionization potential of 21.56 and 40.95 $\mathrm{eV}$ respectively, this ratio traces the massive star content because it is very sensitive to the spectral shape of the UV radiation field. Thornley [10] addressed specifically this issue by modeling the neon ratio as a function of the upper mass cutoff. They used evolutionary synthesis code and photoionisation code to predict the neon line ratio around a cluster of stars. Their results show that a $[\mathrm{Ne} \mathrm{III]/[Ne} \mathrm{II]} \mathrm{line} \mathrm{ratio} \mathrm{of} 0.06$ is consistent with an upper mass cutoff around $25 \mathrm{M}_{\odot}$. However, they found that for shortlived bursts $(1 \mathrm{Myr})$, the measured neon line ratio is consistent with the formation of massive $\left(50-100 \mathrm{M}_{\odot}\right)$ stars.

\footnotetext{
${ }^{1}$ The IRS was a collaborative venture between Cornell University and Ball Aerospace Corporation funded by NASA through the Jet Propulsion Laboratory and the Ames Research Center.
} 
In this paper, we present the spectral map of the $[\mathrm{Ne}$ III]/[Ne II]line ratio. The sensitivity and small aperture size of the IRS gives us the opportunity to do spatial mapping over small scales of the mid-infrared properties of the central region of NGC 253. A total of 45 positions were observed around the bright starburst seen at the center of the galaxy. A spatial gradient is detected in the map.

\section{OBSERVATIONS AND DATA REDUCTION}

The observations were carried out during the science verification phase of the Infrared Spectrograph (IRS) onboard the Spitzer Space Telescope [11] using the Short-High (SH) module [12]. The spectra ranges from 10 to $20 \mu \mathrm{m}$ with a resolution $R \approx 600$. The map is centered at a position of RA $=00^{h} 47^{m} 33.2^{s} \mathrm{DEC}=-25^{\circ} 17^{\prime} 19^{\prime \prime}(\mathrm{J} 2000)$ and the step between each pointing position is $15^{\prime \prime}$ along the length of the slit and $9^{\prime \prime}$ along its width. It spans an area of $700^{\prime \prime} 8 \times 600^{\prime \prime} 8$ at a position angle of 271 degrees. This corresponds to the central $800 \times 688 \mathrm{pc}^{2}$ of the galaxy at the assumed distance of $2.5 \mathrm{Mpc}$. Each slit covers an area of $11 . " 8 \times 5 . ! 3(133 \times 60 \mathrm{pc})$. The exposure time for each slit position was 88 seconds.

The basic data reduction was performed by the standard IRS pipeline version 9.5 at the Spitzer Science Center (SSC). The pipeline removes detector artifacts and cosmic ray signatures and applies the dark and flatfield corrections (Spitzer Observer's Manual ${ }^{2}$, Chapter 7). Standard full-slit spectral extraction and flux calibration were performed as described in the SOM. The uncertainty in the line fluxes is dominated by the absolute flux calibration. At the present state, these uncertainties are on the order of $30 \%$. Uncertainties on the absolute fluxes have little impact on the uncertainties of the relative fluxes. Line ratios are mostly affected by the determination of the continuum near the emission line. In our dataset, this source of error is below $5 \%$.

The $12.8 \mu \mathrm{m}$ [Ne II] and the $15.55 \mu \mathrm{m}$ [Ne III] lines are detected with a signal to noise $(S / N)$ higher than 3, our detection limit, on all the spectra. Line fluxes were measured by fitting a Gaussian profile after subtracting a second order baseline fit.

\section{DISCUSSION}

Figure 1 shows that the $[\mathrm{Ne}$ III]/[Ne II] line ratio increases by a factor $4-6$ from the center of the map toward the edges. This increase is higher than our uncertainties on the relative fluxes. The line ratio at the central pixel is 0.08 , in agreement with what was found with ISO-SWS [9]. The ratio peaks at 0.46 on the south-east edge of the map.

The observed increase of the $[\mathrm{Ne} \mathrm{III}] /[\mathrm{Ne} \mathrm{II}]$ line ratio implies a harder ionization field outside of the nuclear region of NGC 253. In this section, we briefly review the various physical conditions that may lead to an increase of the value of the neon line ratio. We discuss the impact of a metallicity gradient, a modified IMF, an age gradient and the

\footnotetext{
2 http://ssc.spitzer.caltech.edu/documents/som/
} 


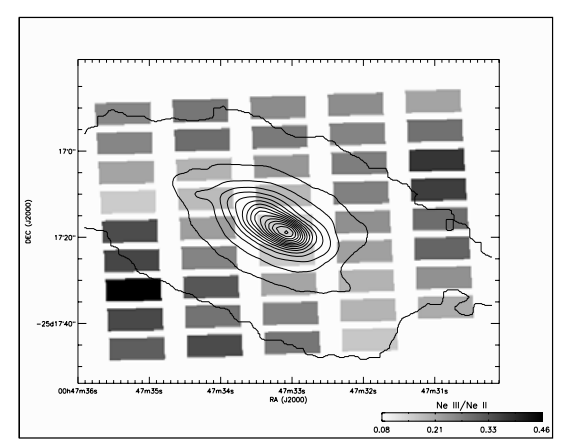

FIGURE 1. Spectral map of the [Ne III]/[Ne II]line ratio over the 2MASS K band contours.

pressure of the ISM on the physical properties of the nuclear region of NGC 253.

A decreasing metallicity leads to a hardening of the ionization field since it affects the line blanketing and the characteristics of the stellar wind [13]. However, a large gradient is needed to account for the observed change. In order to triple the neon line ratio, a change in metallicity close to $1 \mathrm{Z}_{\odot}$ is required [13]. The maps presented here only cover a radius of $0.5 \mathrm{kpc}$ so the implied metallicity gradient needed to reproduce the data is on the order of $2 \mathrm{Z}_{\odot} \mathrm{kpc}^{-1}$.

Several authors $[14,15,5,16,17,18]$ proposed a truncated or steeper IMF to explain the deficiency of massive stars in starburst galaxies. This would imply that the properties of the IMF would change from the nucleus to the outer disk. However, the gradient would imply variations in the IMF only if the stellar populations in the different regions were nearly the same ages.

Aging can cause a softening of the ionization field where a starburst occurs [10]. The observed properties of short-lived starbursts are very sensitive to the massive star content within the first 10 million years of a star formation episode [10]. This means that the hardness of the radiation field, and thus the $[\mathrm{Ne}$ III]/[Ne II] ratio, should decay rapidly if the starburst episode responsible for the ionization field is short-lived. Using this model, our results would indicate that the outside regions are, on average, younger than the nuclear region, consistent with star formation propagating outward.

The pressure of the interstellar medium can indirectly affect the ionization field [19]. If stellar clusters remain buried in their native star formation site, a fair fraction of the UV photons responsible for the ionization of the MIR neon lines will be absorbed. The net effect is a softening of the radiation field. This model would imply that the pressure of the interstellar medium is greater at the central position and decreases outward.

\section{CONCLUSIONS}

A significant gradient in the $[\mathrm{Ne} \mathrm{III}] /[\mathrm{Ne} \mathrm{II}]$ ratio is detected. The ratio rises toward the edges of the map with the lowest value being at the location of the nucleus. To first order, this indicates that the radiation field is harder outside the nucleus which in turn suggests that there is a relative deficiency of massive stars in the nucleus of the galaxy. With 
our dataset, we were able to briefly explore the variation of the ionization field within a single starburst galaxy and compare it with models that are usually compared to the ionization fields of a sample of starburst galaxies. The spectral-mapping capability of the IRS allows detailed studies of the local environment of powerful starbursts and provides unprecedented insight into the star formation process in starbursts. We presented here a small sample of our dataset. A more detailed study on the impact of our observations on the physical properties of the central region of NGC 253 and the stellar population is in preparation.

\section{ACKNOWLEDGMENTS}

This work is based on observations made with the Spitzer Space Telescope, which is operated by the Jet Propulsion Laboratory, California Institute of Technology under NASA contract 1407. Support for this work was provided by NASA through Contract Number 1257184 issued by JPL/Caltech.

\section{REFERENCES}

1. Pence, W. D., ApJ, 239, 54-64 (1980).

2. Jarrett, T. H., Chester, T., Cutri, R., Schneider, S. E., and Huchra, J. P., AJ, 125, 525-554 (2003).

3. Das, M., Anantharamaiah, K. R., and Yun, M. S., ApJ, 549, 896-905 (2001).

4. Scoville, N. Z., Soifer, B. T., Neugebauer, G., Matthews, K., Young, J. S., and Yerka, J., ApJ, 289, 129-140 (1985).

5. Engelbracht, C. W., Rieke, M. J., Rieke, G. H., Kelly, D. M., and Achtermann, J. M., ApJ, 505, 639-658 (1998).

6. Sturm, E., Lutz, D., Tran, D., Feuchtgruber, H., Genzel, R., Kunze, D., Moorwood, A. F. M., and Thornley, M. D., A\&A, 358, 481-493 (2000).

7. Rigopoulou, D., Kunze, D., Lutz, D., Genzel, R., and Moorwood, A. F. M., A\&A, 389, 374-386 (2002).

8. Förster Schreiber, N. M., Sauvage, M., Charmandaris, V., Laurent, O., Gallais, P., Mirabel, I. F., and Vigroux, L., $A \& A$, 399, 833-855 (2003).

9. Verma, A., Lutz, D., Sturm, E., Sternberg, A., Genzel, R., and Vacca, W., A\&A, 403, 829-846 (2003).

10. Thornley, M. D., et al., ApJ, 539, 641-657 (2000).

11. Werner, M. W., et al., ApJS, 154, 1-9 (2004).

12. Houck, J. R., et al., ApJS, 154, 18-24 (2004).

13. Martín-Hernández, N. L., Vermeij, R., Tielens, A. G. G. M., van der Hulst, J. M., and Peeters, E., $A \& A$, 389, 286-294 (2002).

14. Puxley, P. J., Brand, P. W. J. L., Moore, T. J. T., Mountain, C. M., Nakai, N., and Yamashita, T., ApJ, 345, 163-168 (1989).

15. Doyon, R., Joseph, R. D., and Wright, G. S., ApJ, 421, 101-114 (1994).

16. Doherty, R. M., Puxley, P. J., Lumsden, S. L., and Doyon, R., MNRAS, 277, 577-593 (1995).

17. Achtermann, J. M., and Lacy, J. H., ApJ, 439, 163-175 (1995).

18. Beck, S. C., Kelly, D. M., and Lacy, J. H., AJ, 114, 585-591 (1997).

19. Rigby, J. R., and Rieke, G. H., ApJ, 606, 237-257 (2004). 